\title{
What Are the Types of Study Design?
}

\subsection{What Are the Various Types of Clinical Study Designs?}

The quality, reliability, dependability, and publishability of a study depend on its design. A clinical study design includes the preparation of trials, experiments, and observations in research involving human beings. The various types of study designs are depicted in Fig. 8.1.

\subsection{What Are the Types of Observational Studies?}

Broadly there are two types of observational studies, i.e.:

- Descriptive.

- Analytical.

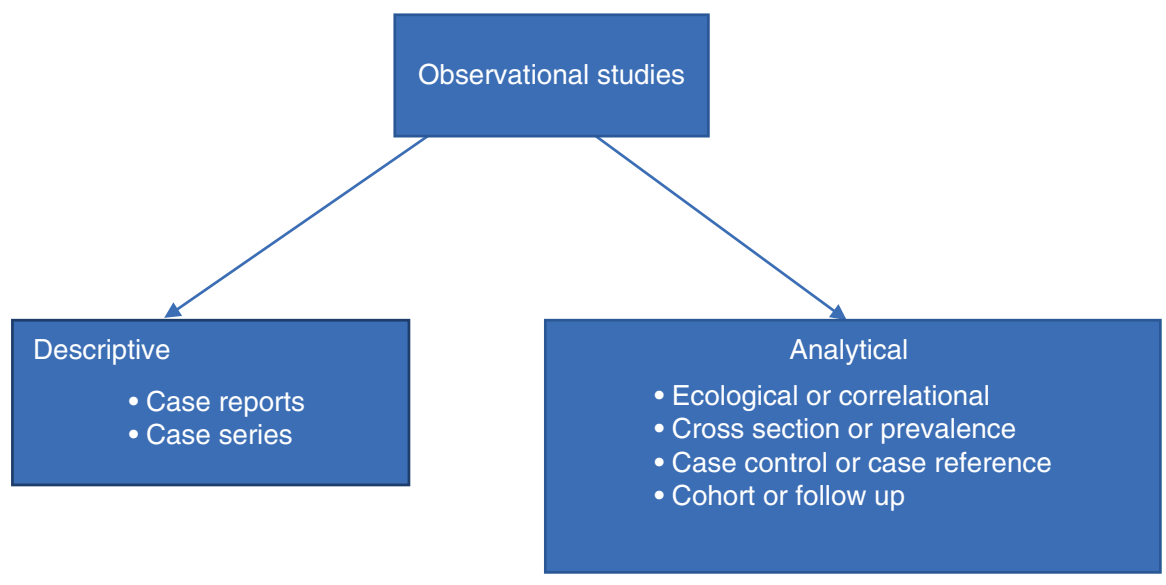

"Education without application is just entertainment."

Tim Sanders, American author and speaker (1959-) 


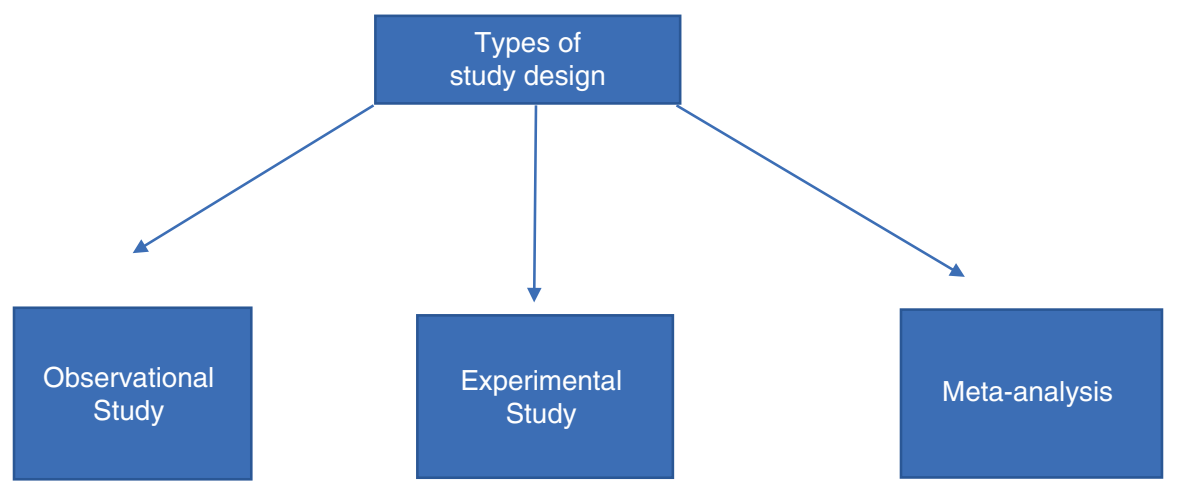

Fig. 8.1 A study can be classified into three major groups: observational, experimental, and meta-analysis

\subsection{What Is a Descriptive Study?}

This kind of study deals with observing the distribution of a given phenomenon. It generally deals with a time, place, and person distribution [1,2].

The procedures involved in a descriptive study include:

- Definition of the population to be studied.

- Naming of the illness.

- Describing the disease by time, place, and person.

- Quantification of the disease outcome.

- Comparing this with known parameters.

The advantages of a descriptive study:

- Provides information regarding the extent of the disease load.

- May suggest a clue to its aetiology.

- Provides background data for planning.

- Contributes to research by describing the illness in relation to time, place, and persons.

Examples of descriptive studies include:

Case reports 1. Profound neutropenia in a patient with COVID-19.

2. Multiple Renal Abscesses in a Horseshoe Kidney

Case series 1. Gastrointestinal manifestations in COVID-19: A review of 30 cases.

2. Long-Term Follow-Ups of Relapses after Surgery for Astrocytoma 


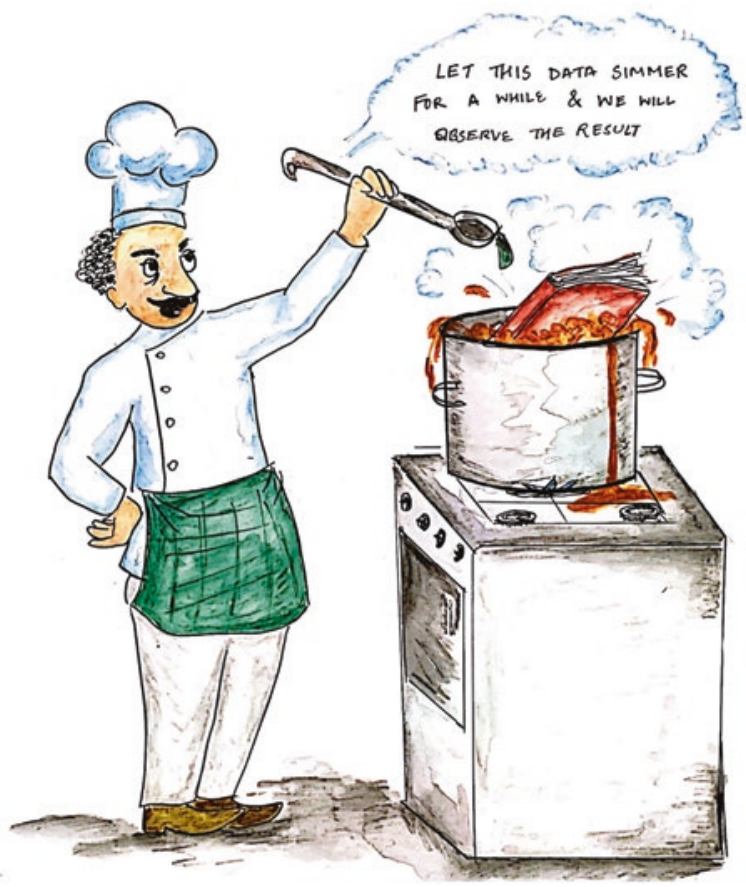

\subsection{What Is Analytical Epidemiology?}

The objective of this kind of study is to test a hypothesis and includes specific subjects of interest. There are four distinct types of investigations:

- Ecological.

- Cross sectional.

- Case control.

- Cohort.

\subsection{What Are Ecological Studies?}

These are observational studies often used to measure the prevalence and incidence of disease, particularly when the disease is rare, and are quite easy to conduct. The other advantage is that they are usually retrospective in nature. In them, there should be only one exposure in the population. An example of such a study would be to compare the prevalence of rheumatoid arthritis in Delhi and Manipur. This data is usually extracted from large databases which may have been used for other purposes and thus are not always reliable. Ecological studies are generally economical and serve as a preliminary point for hypothesis generation [2]. 


\subsection{What Is a Case-Control Study?}

This compares a population with a certain medical condition with another group of people who do not have the disease but are otherwise similar to the study population.

The basic steps include:

- Proper selecting cases and controls.

- Matching of cases with controls.

- Measuring the exposure.

- Analyzing and interpreting the results.

Case-control studies are inexpensive and frequently used kind in epidemiology. Their design allows the study of a rare illness. The preliminary data help to learn what is already known about the association between the risk factors for the disease. The measure of interest is the calculation of the odds ratio. These are also retrospective studies that cannot calculate prevalence and are usually used for rare diseases. They can also be nested within longitudinal studies but given their retrospective nature, can be prone to recall bias [3].

An example of such a study is the occurrence of cervical cancer in patients who have received Human Papillomavirus vaccine in childhood. Figure 8.2 is an example of a case-control study design and how to calculate the odd's ratio.

\subsection{What Is a Cohort Study?}

A cohort study is done on a group of people who are followed up over many yearsfor instance, to determine how often a certain disease occurs. It is performed to obtain evidence to support the existence of an association between a suspected cause and disease (Fig. 8.3).

Types of cohort study:

- Prospective cohorts.

- Retrospective cohorts.

- Combination of prospective and retrospective cohorts.

Elements of a cohort study:

- Collection of study patients.

- Procuring data on exposure.

- Study of comparison groups.

- Review visits.

- Final data analysis.

These studies can help in calculating point prevalence or period prevalence. Prospective cohort studies are the 'gold standard for observational research'. 


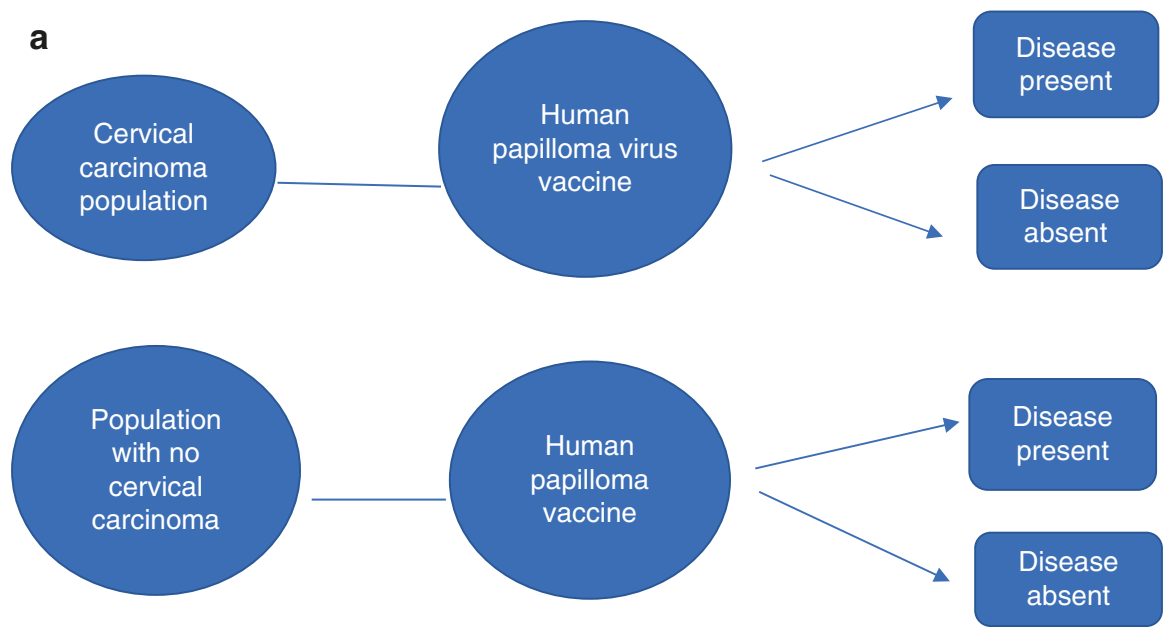

b

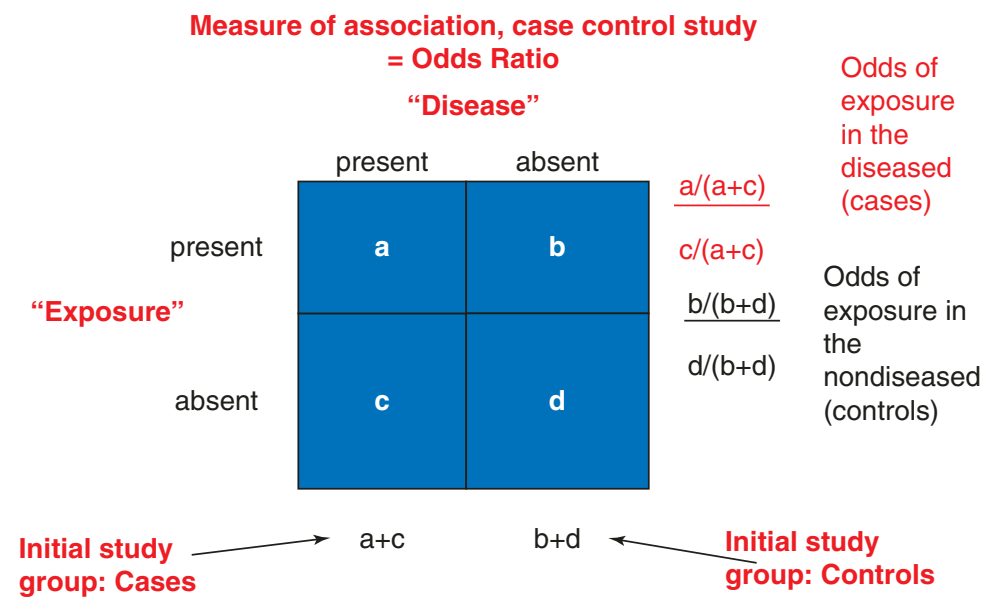

Fig. 8.2 (a, b) Case-control study and calculation of odds ratio

\subsection{What Are Cross-Sectional Studies?}

These are also retrospective and study the prevalence of a disease. They are economical and easy to conduct. An example of a cross-sectional study design would be enrolling participants who are either current alcohol consumers or have never consumed alcohol, and are being assessed whether or not they have liver-related issues. The studies assess both exposure and outcome at a single point in time. Figure 8.4 shows an example of this $[3,4]$. 


\section{Past}

Present

Future

Start study

\section{Retrospective cohort study}

\begin{tabular}{|c|c|}
$\begin{array}{c}\text { Composition of } \\
\text { cohort and } \\
\text { assessment } \\
\text { of exposure }\end{array} \longrightarrow \begin{array}{c}\text { Assessment of } \\
\text { outcome in } \\
\text { exposed and } \\
\text { unexposed group }\end{array}$ \\
\hline
\end{tabular}

Prospective cohort study

\begin{tabular}{|c|c|}
$\begin{array}{c}\text { Composition of } \\
\text { cohort and } \\
\text { assessment } \\
\text { of exposure }\end{array} \longrightarrow \begin{array}{c}\text { Assessment of } \\
\text { outcome in } \\
\text { exposed and } \\
\text { unexposed group }\end{array}$ \\
\hline
\end{tabular}

Fig. 8.3 Depicts both a retrospective and a prospective cohort study

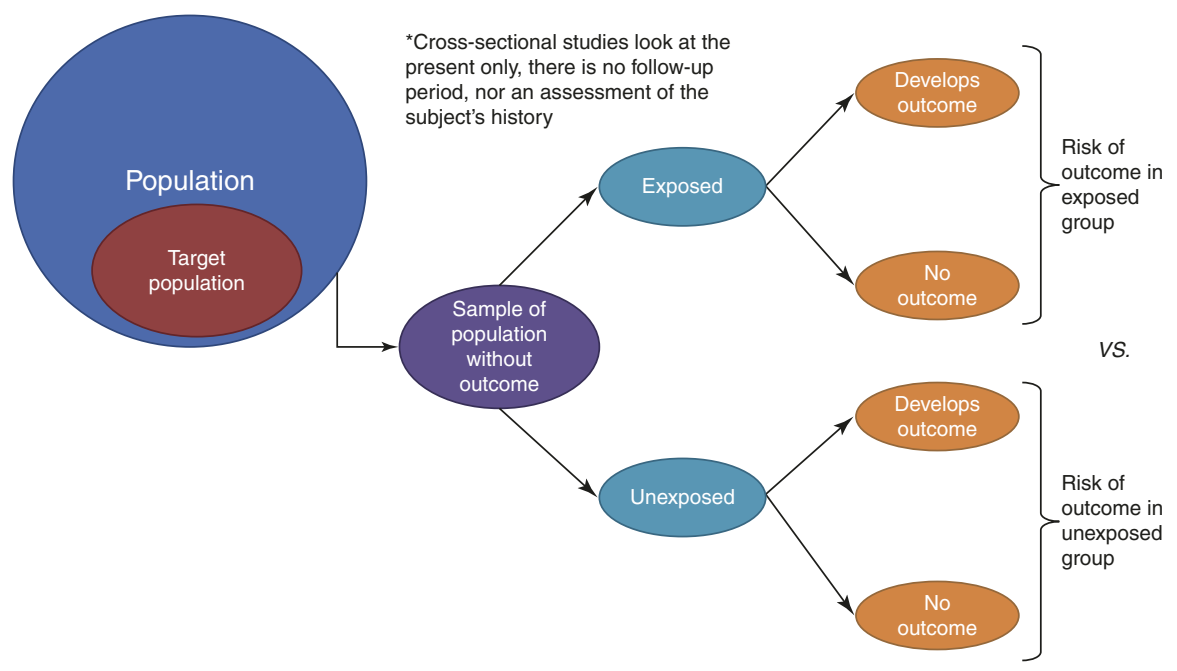

Fig. 8.4 Cross-sectional study

\subsection{What Is an Experimental Study Design?}

This has a similar approach to a cohort study except that it is carried out under direct control of an investigator. The aim is to provide systematic proof of either aetiological or risk factors of the disease the modification of which can control it. Epidemiological and interventional research studies include three elements: 
1. Definition and measure of exposure in two or more groups.

2. Measure of disease outcome(s) in the same groups.

3. Statistical comparison made between groups to assess potential relationships between the exposure and outcome, all of which are defined by the researcher.

\subsection{What Is a Randomized Controlled Trial?}

This is a study performed to avoid any bias while testing for the efficacy of, e.g., a drug. The study population is randomly divided into two groups, of which one receives the drug under study and the second group receives a placebo and acts as the control group. The experiment may be blinded, which means that any information which may influence the participant is withheld while the trial is ongoing or maybe double blinded in which the information is withheld from both the subject and the investigator [5].

The basic steps of a randomized control trial (RCT) include:

- Writing a protocol.

- Selecting a normal and experimental population.

- Randomization.

- Intervention in the study group and placebo.

- Follow up.

- Measuring the outcome of interest.

\subsection{Design of a Randomized Control Trial (Fig. 8.5)}

\subsection{What Are the Standards of Research and Reporting?}

There are many available guidelines on study design, execution, and how it needs to be reported in the final manuscript. This improves the quality of a research paper and allows results to be presented in a systematic manner for a sound conclusion to be drawn. Table 8.1 mentions some important reporting formats and their websites. 


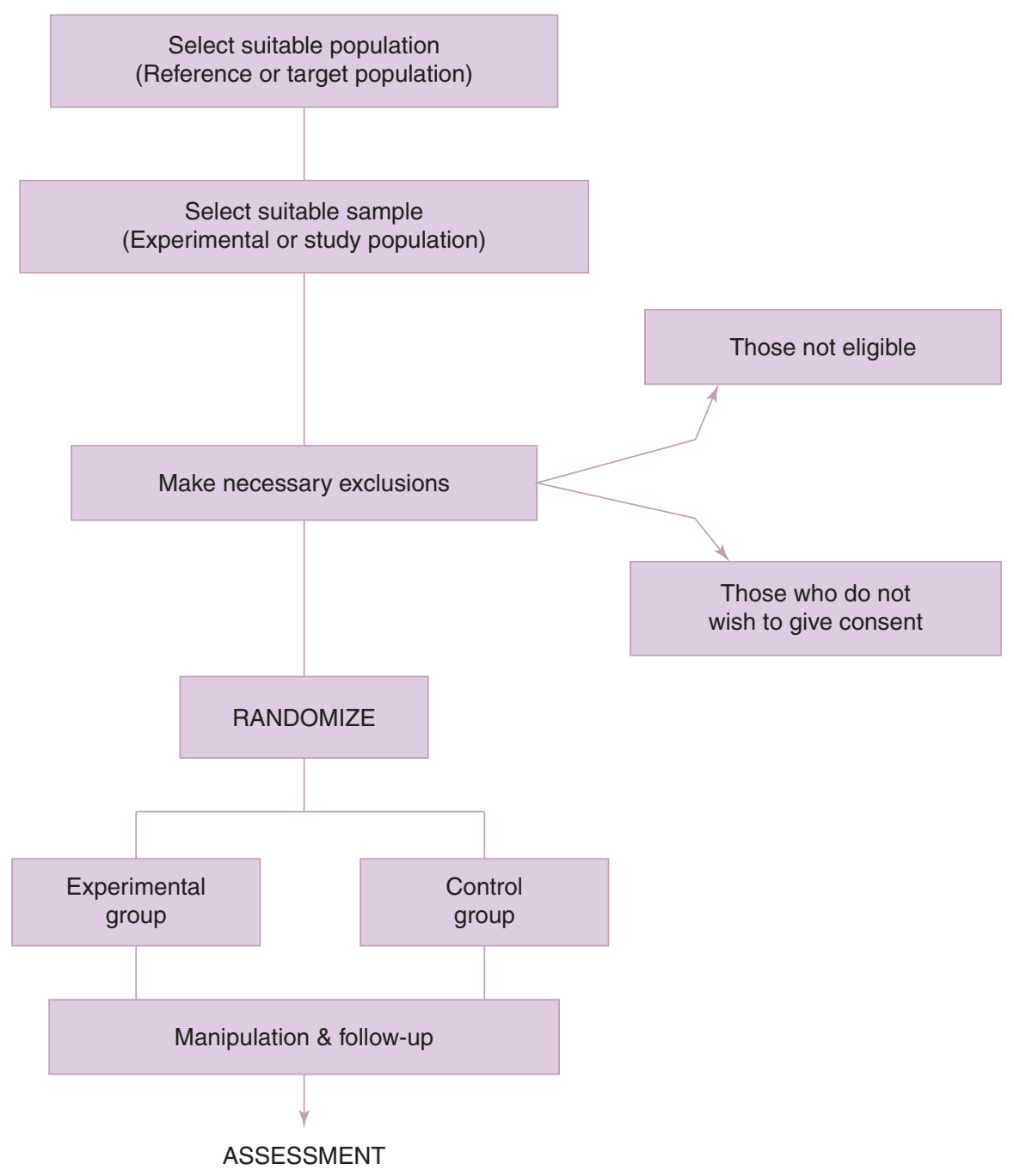

Fig. 8.5 Randomized control trial

Table 8.1 Published standards for study design and reporting

\begin{tabular}{l|l|l}
\hline Study design & Abbreviation & Website \\
\hline $\begin{array}{l}\text { Randomized controlled trials } \\
{[6]}\end{array}$ & CONSORT & http://www.consort-statement.org/downloads \\
\hline Systematic reviews [7] & PRISMA & http://www.prisma-statement.org/ \\
\hline Observational studies [8] & STROBE & https://www.strobe-statement.org/home \\
\hline Case reports [9] & CARE & https://www.care-statement.org/ \\
\hline Qualitative research [10] & COREQ & https://academic.oup.com/ \\
\hline
\end{tabular}




\subsection{Conclusions}

- Formulating a study design is the most important part of the planning stage of clinical research. It is an indispensable part of new drug discovery.

- Basic research is also called experimental and done in genetics, biochemistry, and physiology. Studies on drug properties are also included in this.

- Clinical studies can be interventional or non-interventional. Interventional studies are done on surgery, chemotherapeutic agents, devices, or drugs.

- A rare disease is best investigated by a case-control study and rare exposures by cohort studies.

- A retrospective study is based on historical data, which may be obtained from past records. In prospective studies the data are collected after the work has begun.

- Observational studies are divided into descriptive and analytical studies.

- In cohort studies, two or more groups are selected on the basis of their exposure to a drug or environmental exposure and then followed up for outcome.

- The evidence collected from randomized controlled trials is of good quality. They allow a proper evaluation of a drug. More recently adaptive designs allow for greater flexibility and pragmatic randomized trials.

\section{References}

1. Aggarwal R, Ranganathan P. Study designs: part 2 - descriptive studies. Perspect Clin Res. 2019;10(1):34-6.

2. Thiese MS. Observational and interventional study design types; an overview. Biochem Med (Zagreb). 2014;24:199-210.

3. Anglemyer A, Horvath HT, Bero L. Healthcare outcomes assessed with observational study designs compared with those assessed in randomized trials. Cochrane Database Syst Rev. 2014;4:MR000034.

4. Centers for disease control and prevention. Descriptive and analytic studies. Last accessed on 20th April 2020. Available on https://www.cdc.gov/globalhealth/healthprotection/fetp/training_modules/19/desc-and-analytic-studies_ppt_final_09252013.pdf.

5. Kendall JM. Designing a research project: randomised controlled trials and their principles. Emergency Med J. 2003;20:164-8.

6. CONSORT-transparent reporting of trials. Lasts accessed on 20th April 2020. Available on http://www.consort-statement.org/downloads.

7. PRISMA- Transparent reporting of systematic reviews and meta analyses. Lasts accessed on 20th April 2020. Available on http://www.prisma-statement.org/.

8. STROBE statement- strengthening the reporting of observational studies in epidemiology. Lasts accessed on 20th April 2020. Available on https://www.strobe-statement.org/home.

9. CARE- case report guidelines. Lasts accessed on 20th April 2020. Available on https://www. care-statement.org/.

10. OXFORD academic. Lasts accessed on 20th April 2020. Available on https://academic.oup.com 
Open Access This chapter is licensed under the terms of the Creative Commons Attribution 4.0 International License (http://creativecommons.org/licenses/by/4.0/), which permits use, sharing, adaptation, distribution and reproduction in any medium or format, as long as you give appropriate credit to the original author(s) and the source, provide a link to the Creative Commons license and indicate if changes were made.

The images or other third party material in this chapter are included in the chapter's Creative Commons license, unless indicated otherwise in a credit line to the material. If material is not included in the chapter's Creative Commons license and your intended use is not permitted by statutory regulation or exceeds the permitted use, you will need to obtain permission directly from the copyright holder. 